\title{
The Use of Coding and Diversity Combining for Mitigating Fading Effects in a DS/CDMA System
}

\author{
Pilar Díaz, Member, IEEE, and Ramón Agustí, Member, IEEE
}

\begin{abstract}
Spatial diversity is an attractive technology to cope with the fading channel encountered in mobile communications. This paper presents novel closed analytical expressions of the bit-error rate (BER) achievable in a coherent binary phase-shiftkeying (CBPSK) direct-sequence code-division multiple-access (DS/CDMA) system for any power delay profile and for either postdetection selection or maximal ratio combining (MRC). In particular, expressions for the cutoff rate $R_{o}$ and for its related parameter $D$ are also formulated in order to assess the system performance under the consideration of some channel coding schemes. Finally, an exemplary study is carried out in order to illustrate the behavior of a realistic space-diversity code-division multiple-access (CDMA) system according to the analytical expressions that have been derived.
\end{abstract}

Index Terms - Channel coding, code-division multiple access, fading channel, mobile communications, spatial diversity.

\section{INTRODUCTION}

$\mathbf{T}$ HE ADVANTAGES of using spatial diversity have been widely recognized in the code-division multiple-access (CDMA) field, although not much effort has been devoted so far in the open literature to assess analytically these techniques. In this paper, closed analytical expressions of the bit-error rate (BER) achievable with antenna diversity and ideal averaged power control are presented for a coherent binary phaseshift-keying (CBPSK) direct-sequence code-division multipleaccess (DS/CDMA) system with a RAKE receiver in the presence of generic multipath Rayleigh fading. This might model rather well the CDMA system for high fading rates since, in practice, closed power control, as it is usually invoked in CDMA systems to mitigate the fast fading, loses effectiveness for high Doppler rates $\left(f_{m}\right)$. Studies shown in [1] and [2] reveal that moderate $f_{m}$ values only lead to moderate reductions on compensating the fast fading. That is, the fading statistics of the signal after closed power control remains nearly the same that those obtained with only averaged power control. In particular, for $f_{m}=80 \mathrm{~Hz}$, as much as an $87 \%$ of the variability is maintained [1].

The analysis pursued in this work includes both selection and maximal ratio combining (MRC) of the outputs of $P$ Rake

Manuscript received September 30, 1994; revised July 1, 1996. This work was supported by CICYT (Spanish Education Ministry) under Contract TIC940870.

The authors are with the Department of Signal Theory and Communications, Universitat Politècnica de Catalunya, 08034 Barcelona, Spain (e-mail: pilar@xaloc.upc.es).

Publisher Item Identifier S 0018-9545(98)00688-4. receivers corresponding to $P$ different antennas. In particular, MRC is well suited as a form of microdiversity combining at the same site, while selection could be suited as a form of macrodiversity combining to be realized on the network side. In this case, every base station involved (usually, a total of two) receives the signal from one mobile. At a higher level in the network hierarchy, which belongs to the access network to the mobile interworking unit [3], only the base station with the best signal-to-interference ratio at the Rake output is selected. Moreover, the selection diversity performance allows us to have an analytical upper bound of the performance (in terms of BER) of the switching microdiversity scheme [4], [5]. This simple scheme uses $P$ antennas, but only one RF front end and one Rake receiver, so that when the signal level at the output of the Rake crosses down a preestablished threshold, the input of the receiver is switched to another antenna.

No coding as well as convolutional channel coding schemes have also been considered. In the case of coding, closed expressions of the channel cutoff rate have been formulated in the presence of ideal interleaving (infinite depth), so that available bounds of the BER in terms of the channel cutoff rate $R_{0}$ can be used. In every case, as usually has been done in the related literature, the Gaussian model for interferences has been assumed.

The organization of this paper is as follows. Section III is devoted to assess BER expressions of a DS/CDMA system in the presence of Rayleigh fading under diversity combining. Both selection and MRC are considered as diversity schemes. In Section IV, the use of channel coding is also contemplated and the parameter $D$, which appears in the BER bounds for convolutional codes, is derived for soft decisions. Section $\mathrm{V}$ is devoted to analyze and compare the performance of both diversity schemes in conjunction with two different convolutional codes. Finally, in Section VI, some conclusions are drawn.

\section{SYSTEM MODEL}

CDMA is a digital multiple-access technique in which each subscriber has his own identification code and all the transmitted signals share the same frequency band of the spectrum. The identification code is an $N$-long pseudorandom binary sequence, unique for each active user. This sequence is made up of $N$ waveforms, called chips, the duration of which is $T_{c}$. The code length expressed in time is $T_{b} \mathrm{~s}$, so $T_{b}=N T_{c}$. 


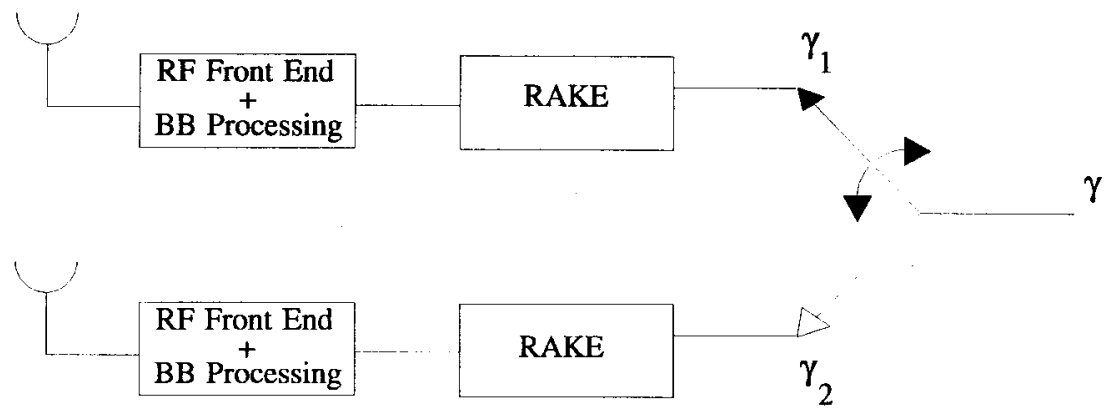

Fig. 1. Selection diversity of order 2 at reception.

The low-pass equivalent impulse response for the link between an active user and the base station is assumed to have $L$ propagation paths

$$
h(t)=\sum_{n=0}^{L-1} \alpha_{n} e^{j \theta_{n}} \delta\left(t-n T_{c}\right)
$$

where the path amplitude $\alpha_{n}$ is assumed to be a Rayleigh random variable and the path phase $\theta_{n}$ a uniform random variable within $[0,2 \pi]$.

The optimum receiver of a DS/CDMA system operating in such a kind of environments, called the Rake receiver [6], has also been considered in order to take advantage of the multipath propagation, characteristic in mobile communications. This receiver recovers the information carried by any propagation path since it has available $L$-transmitted signal replicas. However, when the number of propagation paths $L$ is so large that the receiver complexity increases excessively, or when the signal power received via the last replicas is negligible with regard to that of the first ones, the Rake receiver only combines the received signals from $M$ (out of $L$ ) different propagation paths.

Furthermore, the presence of deep fades, characteristic in Rayleigh fading channels, produce high error rates. To counteract the effects of these propagation conditions, one may resort to employ antenna diversity in conjunction with the multipath diversity provided by the Rake receiver.

\section{Bit-ERror Probability Under Diversity COMBINING}

Closed expressions of the bit error probability have been derived for a coherent BPSK DS/CDMA system under Rayleigh multipath fading when $P$ antennas are employed at the receiver. Selection diversity and MRC have been considered and compared as different form of diversity.

1) Selection Diversity: Fig. 1 shows the receiver structure for the particular case of selection diversity with two antennas at reception. The receiver contains the RF front end, a baseband processing module, and the Rake combiner after the output of each antenna.

$\gamma_{i}$ denotes the overall signal-to-interference ratio at the output of the Rake corresponding to the $i$ th antenna and is given by [6]

$$
\gamma_{i}=\sum_{k=0}^{M-1} \gamma_{k i}
$$

where $M$ is the number of arms in the Rake and $\gamma_{k i}$ ( $i=$ $1, \cdots, P)$ is the signal-to-interference ratio of the $k$ th path in the $i$ th antenna. When selection is employed as form of diversity, the signal-to-interference ratio $\gamma$ before decision is given by

$$
\gamma=\max \left(\gamma_{1}, \cdots, \gamma_{P}\right)
$$

For coherent BPSK signaling, the averaged BER can be expressed as

$$
P_{e}=E\left[\frac{1}{2} \operatorname{erfc}(\sqrt{\gamma})\right] .
$$

In order to assess the expression of $P_{e}$, the probability density function (pdf) of $\gamma$ must be determined. It can be obtained from the pdf of $\gamma_{1}, \cdots, \gamma_{P}$, which are given by [6]

$$
f_{\gamma_{i}}=\sum_{k=0}^{M-1} \frac{\pi_{k i}}{\bar{\gamma}_{k i}} \exp \left(-\frac{\gamma_{i}}{\bar{\gamma}_{k i}}\right), \quad i=1, \cdots, P
$$

in the presence of different Rayleigh fadings. $\bar{\gamma}_{k i}$ is the signalto-interference ratio of every path, and the coefficients in the above expression $\pi_{k i}$ can be expressed as

$$
\pi_{k i}=\prod_{\substack{j=0 \\ j \neq k}}^{M-1} \frac{\bar{\gamma}_{k i}}{\bar{\gamma}_{k i}-\bar{\gamma}_{j i}}, \quad i=1, \cdots, P .
$$

Therefore, the pdf of $\gamma$ can be expressed as

$$
\begin{aligned}
f_{\gamma}(\gamma)= & \sum_{i=1}^{P} F_{\gamma_{i}}(\gamma) f_{\gamma_{i}}(\gamma) \\
= & \sum_{i=1}^{P} \sum_{n=0}^{M-1} \pi_{n i}\left[1-\exp \left(-\frac{\gamma}{\bar{\gamma}_{n i}}\right)\right] \\
& \cdot \sum_{k=0}^{M-1} \frac{\pi_{k i}}{\bar{\gamma}_{n i}} \exp \left(-\frac{\gamma}{\bar{\gamma}_{n i}}\right)
\end{aligned}
$$

where $F_{x}(x)$ represents the distribution function of the random variable $x$. From the expression of the pdf of $\gamma$, it is possible to compute the mean BER given by (4) for coherent BPSK and Rayleigh fading with antenna diversity. The expression for the BER, which has been derived in detail in Appendix 


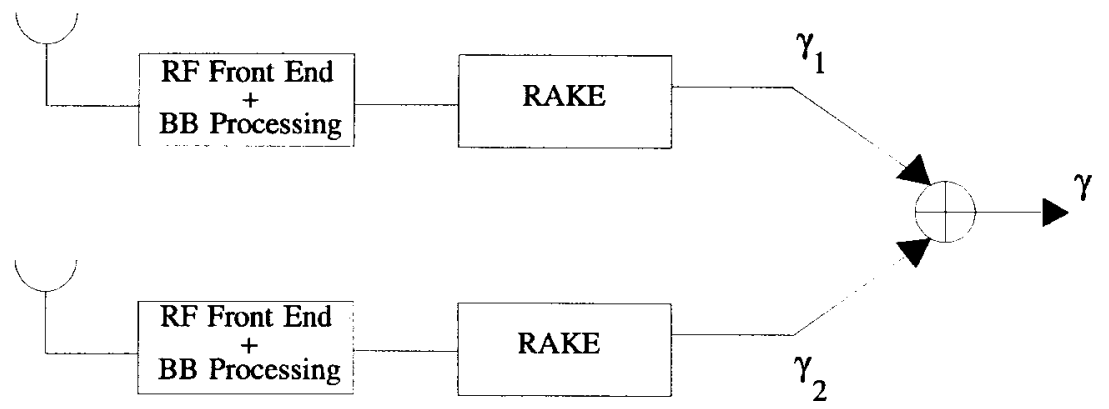

Fig. 2. MRC diversity of two at reception.

$\mathrm{A}$, is as follows:

$$
\begin{aligned}
P_{e}= & \frac{1}{2} \sum_{i=1}^{P} \sum_{n=0}^{M-1} \sum_{k=0}^{M-1} \pi_{n i} \pi_{k i} \\
& \cdot\left[\frac{\bar{\gamma}_{k i}}{\bar{\gamma}_{k i}+\bar{\gamma}_{n i}}+\frac{\bar{\gamma}_{n 1}}{\bar{\gamma}_{k i}+\bar{\gamma}_{n i}}\right. \\
& \cdot \sqrt{\frac{\bar{\gamma}_{k i} \bar{\gamma}_{n i}}{\bar{\gamma}_{k i}+\bar{\gamma}_{n i}+\bar{\gamma}_{k i} \bar{\gamma}_{n i}}}-\sqrt{\left.\frac{\bar{\gamma}_{k i}}{1+\bar{\gamma}_{k i}}\right]}
\end{aligned}
$$

2) MRC Diversity: Fig. 2 shows the receiver structure for the particular case of MRC diversity with two antennas at reception, analogously to Fig. 1 for selection diversity.

When MRC is employed as form of diversity, $\gamma$ before decision can be expressed as

$$
\gamma=\sum_{i=1}^{P} \gamma_{i}=\sum_{i=1}^{P} \sum_{k=0}^{M-1} \gamma_{k i}
$$

where $\gamma_{i}$ is again given by (2).

The expression of the mean BER is (4) for coherent BPSK signaling, so the pdf of $\gamma$ must be determined in order to assess $P_{e}$. It can be done by means of its characteristic function, which can be expressed as

$$
\psi_{\gamma}=\prod_{k=0}^{M-1} \prod_{i=1}^{P} \frac{1}{1-j \omega \bar{\gamma}_{k i}}
$$

since the random variables $\gamma_{k i}(i=1, \cdots, P$ and $k=$ $0, \cdots, M-1)$ are statistically independent. Therefore, the pdf of $\gamma$ is determined from the inverse Fourier transform of $\psi_{\gamma}$ given by

$$
f_{\gamma}(\gamma)=\sum_{k=0}^{M-1} \sum_{i=1}^{P} \frac{\pi_{k i}}{\bar{\gamma}_{k i}} \exp \left(-\frac{\gamma}{\bar{\gamma}_{k i}}\right)
$$

provided that $\bar{\gamma}_{k i}$ have different values. The parameters $\pi_{k i}$ are given by (6). In such a case, the expression of the mean BER in presence of Rayleigh fading can be directly derived from [6]

$$
P_{e}=\frac{1}{2} \sum_{k=0}^{M-1} \sum_{i=1}^{P} \pi_{k i}\left[1-\sqrt{\frac{\bar{\gamma}_{k i}}{1+\bar{\gamma}_{k i}}}\right] .
$$

When $\bar{\gamma}_{k 1}=\bar{\gamma}_{k 2}=\cdots=\bar{\gamma}_{k P}(k=0, \cdots, M-1)$, the above expression for the mean BER is no longer valid, and in this case, the characteristic function is expressed as

$$
\psi_{\gamma}=\prod_{k=0}^{M-1} \frac{1}{\left(1-j \omega \bar{\gamma}_{k 1}\right)^{P}} .
$$

Only for $P=2$ it is possible to derive a simple and closed analytical expression for the pdf of variable $\gamma$, which is computed as the inverse Fourier transform of $\psi_{\gamma}$ and given by

$$
\begin{aligned}
f_{\gamma}(\gamma)= & \sum_{k=0}^{M-1} \pi_{k 1}\left[\frac{\gamma}{\bar{\gamma}_{k 1}^{2}}-\frac{2}{\bar{\gamma}_{k 1}} \sum_{j \neq k} \frac{\bar{\gamma}_{j 1}}{\bar{\gamma}_{k 1}-\bar{\gamma}_{j 1}}\right] \\
& \cdot \exp \left(-\frac{\gamma}{\bar{\gamma}_{k 1}}\right)
\end{aligned}
$$

From (14), it is possible to determine again the mean BER for coherent BPSK with Rayleigh fading and antenna MRC diversity. The expression of the mean BER, which has been derived in detail in Appendix B, is as follows:

$$
\begin{aligned}
P_{e}=\sum_{k=0}^{M-1} \pi_{k 1} & {\left[\left(\frac{1-\mu_{k 1}}{2}\right)^{2}\left(2+\mu_{k 1}\right)\right.} \\
& \left.-\left(1-\mu_{k 1}\right) \sum_{j \neq k} \frac{\bar{\gamma}_{j 1}}{\bar{\gamma}_{k 1}-\bar{\gamma}_{j 1}}\right]
\end{aligned}
$$

where

$$
\mu_{k 1}=\sqrt{\frac{\bar{\gamma}_{k 1}}{1+\bar{\gamma}_{k 1}}}
$$

\section{CODING With SofT DECISIONS}

In mobile radio communication systems, there exists the need to employ forward-error-correction (FEC) techniques in order to protect the information against the severity of the multipath propagation. Moreover, it is well known that in spread-spectrum systems, as CDMA systems are, the use of channel coding does not require additional allocation of bandwidth [7]. In case of using convolutional codes as well as decoders that work with soft decisions at their inputs, bounds on the BER may be obtained by resorting to the expression [8]

$$
P(b) \leq G(D)
$$


where $G($.$) is a function determined solely by the specific code,$ whereas the parameter $D$ depends only on the coding channel and the decoder metric.

The use of this bound causes the parameter- $D$ appearance, which is related to the cutoff rate $R_{0}$ as follows [8]:

$$
D=2^{-R_{o}+1}-1
$$

and is defined as

$$
\begin{gathered}
D=\min _{\lambda \geq 0} D(\lambda) \\
\text {. }
\end{gathered}
$$

where

$$
D(\lambda)=E\left[e^{\lambda r\left(x^{*}-x\right)} \mid x\right] .
$$

In the above expression, $x$ and $x^{*}$ represent two different encoded sequences, and $r$ is the received sequence when $x$ has been the transmitted one. The parameter $D$ does not depend on the code, but on the channel, modulation scheme, receiver, and also on the kind of decisions employed by the decoder, that is, hard or soft decisions. Therefore, in order to assess the BER as a function of the capacity, it is necessary to derive the expression of the parameter $D$ as shown below.

When the decoder works with hard decisions, $D$ depends directly on the BER of the channel, and is given by [9]

$$
D=\sqrt{4 P_{e}\left(1-P_{e}\right)}
$$

where $P_{e}$ is the average BER at the decoder input.

When it works with soft decisions, $D$ depends on the channel statistics and transmission schemes. In particular, when selection diversity with $P$ antennas having the same signal-to-interference ratio for paths with identical delays is employed in a Rayleigh multipath channel, the expression of $D$ for coherent BPSK, which has been given in detail in Appendix $\mathrm{C}$, is as follows:

$$
\begin{aligned}
D= & P \sum_{n=0}^{M-1} \sum_{k=0}^{M-1} \pi_{n 1} \pi_{k 11} \\
& \cdot\left[\frac{1}{1+\bar{\gamma}_{k 1}}-\frac{\bar{\gamma}_{n 1}}{\bar{\gamma}_{n 1}+\bar{\gamma}_{k 1}+\bar{\gamma}_{n 1} \bar{\gamma}_{k 1}}\right]
\end{aligned}
$$

where $\pi_{n 1}$ and $\pi_{k 1}$ are given by (6). On the other hand, when MRC is selected as form of diversity, the parameter $D$ can be expressed as (see Appendix D)

$$
D=\prod_{n=0}^{M-1}\left(\frac{1}{1+\bar{\gamma}_{n 1}}\right)^{2} .
$$

\section{System PERFormanCE}

In order to illustrate the behavior of a space-diversity-based CDMA system according to the expressions obtained above and show how to assess the performance of such a system in a given environment, we have first computed from (8) and (15) the mean BER of a two-antenna diversity DS/CDMA cellular system in a Rayleigh multipath environment with the same statistics in both antennas.

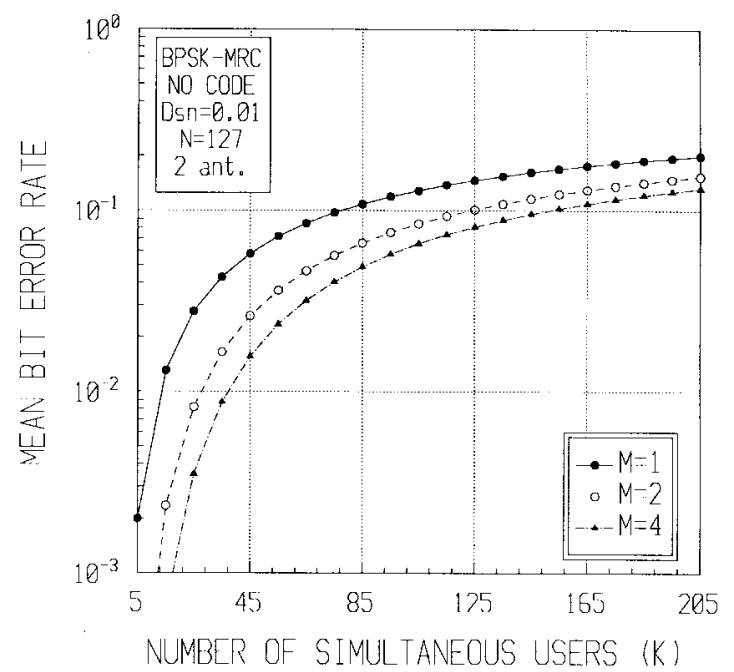

Fig. 3. Mean BER for selection diversity with two antennas at the receiver.

To compare the different diversity combining schemes, let us assume the power delay profile (PDP) of the channel $p(\tau)$ to be exponential [11] and identical for any antenna

$$
p(\tau)=\frac{1}{D_{s}} \exp \left(-\frac{\tau}{D_{s}}\right)
$$

where $D_{s}$ denotes the delay spread of the channel. In this situation, the variance of $\alpha_{k i}$, which represents the complex Gaussian amplitude of the $k$ th propagation path between the transmitter and the $i$ th antenna at reception, is given by

$$
\bar{\alpha}_{k i}^{2}=p\left(k T_{c}\right)
$$

where $T_{c}$ is the chip interval.

The mean signal-to-interference ratio of the $k$ th path at the $i$ th antenna can be formulated as [10]

$$
\bar{\gamma}_{k i}=\frac{\bar{\alpha}_{k i}^{2}}{\frac{2 \rho K}{3 N} \sum_{j=0}^{L-1} \bar{\alpha}_{j i}^{2}}
$$

where $K$ is the number of simultaneous users of the system within the cell of interest, $\rho$ is the reuse factor that takes into account interference from other cells, $N$ is spread-spectrum processing gain, and $L$ the total number of arrival paths at the receiver.

Furthermore, in order to assess the performance of the system for different levels of complexity in the Rake receiver, which is actually built up with $M$ arms, we have assumed representative values for the parameters on the expressions derived for the mean BER, as shown below:

1) delay spread normalized to the bit period $T_{b}: D_{s n}=$ $D_{s} / T_{b}=0.01$

2) processing gain: $N=127$;

3) reuse factor [12]: $\rho=1.7$.

Figs. 3 and 4 show the mean BER for coherent BPSK of a DS/CDMA cellular system that make use of two antennas at reception with selection and MRC diversity, respectively, and in particular, for the environment described above. 
TABLE I

BE of a DS/CDMA System with CBPSK And Channel Coding ( $r-1 / 3$ Convolutional Code and Hard Decisions)

\begin{tabular}{c|c|c|c}
\hline \hline M & No Diversity & Selection Div. & MRC Diversity \\
\hline 1 & 0.07 & 0.16 & 0.21 \\
\hline 2 & 0.15 & 0.25 & 0.38 \\
\hline 4 & 0.21 & 0.31 & 0.49 \\
\hline
\end{tabular}

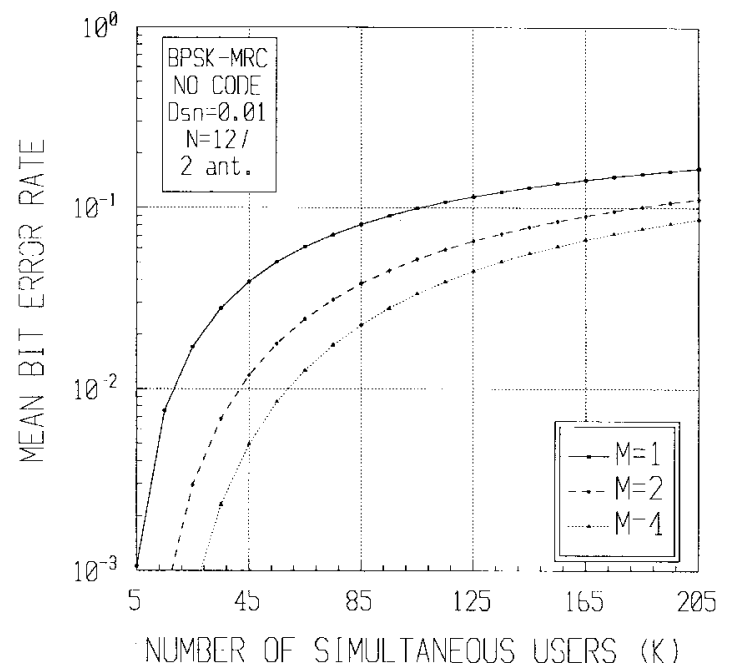

Fig. 4. Mean BER for MRC diversity with two antennas at the receiver.

Concerning channel coding, two representative convolutional codes have been considered. Bounds on the mean BER for each one of them, derived from the Chernoff bound, are given by [9].

1) $r-1 / 2$ convolutional code with generator polynomial 133 171 (octal)

$$
P_{b} \leq \frac{1}{2}\left(36 D^{10}+211 D^{12}+1404 D^{14}+11633 D^{16}\right)
$$

2) $r-1 / 3$ convolutional code with generator polynomial 133 145175 (octal)

$$
P_{b} \leq \frac{1}{2}\left(7 D^{15}+8 D^{16}+22 D^{17}+44 D^{18}\right) .
$$

In order to compare the different schemes, the system performance has been evaluated in terms of the bandwidth efficiency, which is defined as the number of bits simultaneously transmitted by $K$ users divided by the total available bandwidth and which is given by [10]

$$
B E=\frac{K r}{N}
$$

where $r$ is the coding rate.

To assess the preciseness of the analytical results obtained from the derived expressions of the mean BER, they have been compared with results obtained from simulations. To do that, an identical channel to that assumed to derive the analytical expressions has been simulated, ideal averaged power control has been assumed and the $r-1 / 3$ convolutional code has been taken as representative. The encoder and decoder of the

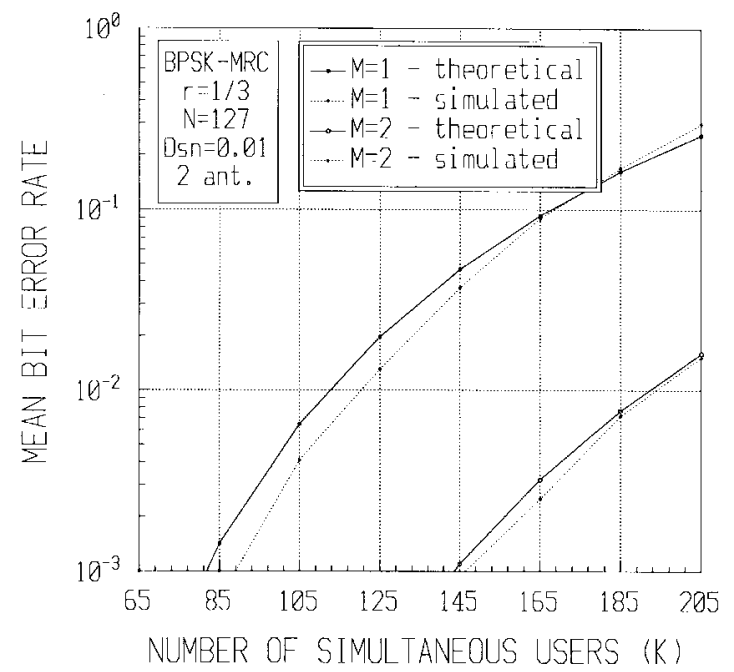

Fig. 5. Mean BER for MRC diversity with a $r-1 / 3$ convolutional code.

convolutional code have also been simulated in order not only to evaluate the preciseness of the obtained analytical expressions, but also the bounds given in (27) and (28). The theoretical results and those obtained in the simulations are depicted in Fig. 5. The curves shown in this figure are so close for both $M=1$ and $M=2$ that it can be stated that the upper bounds on the mean BER for convolutional codes are very tight, except when the channel introduces too many errors $\left(P_{b}>10^{-1}\right)$ so that the convolutional decoder significantly degrades and bounds on the BER are no longer valid. Moreover, when $M$ increases the bounds on the BER tend to be tighter as it is expected.

Table I shows the BE for a DS/CDMA cellular system with no diversity (only one antenna), selection diversity, and MRC diversity (two antennas). It has been computed for the value of $K$ that supports a transmission with a mean BER $=10^{-3}$. In this case, the $r-1 / 3$ convolutional code with hard decisions has been considered as the channel coding employed in the system. In examining the BE values shown in Table I, it is apparent that diversity significantly improves capacity.

Table II shows the BE for a DS/CDMA system with selection diversity for both convolutional codes $(r=1 / 2$ and $r=1 / 3$ ) with hard and soft decisions and Table III for MRC diversity. In both cases, selection or MRC, the BE is nearly twice greater when the decoder works with soft decisions, and as a result, the capacity may be increased in the same way.

In all the tables shown above, it can also be noticed the capacity gain in terms of receiver complexity by means of the parameter $M$. 
TABLE II

BE of a DS/CDMA System with CBPSK and Channel Coding for Selection Diversity

\begin{tabular}{c|c|c|c|c}
\hline \hline$M$ & $\mathrm{r}=1 / 2$ hard & $\mathrm{r}=1 / 2$ soft & $\mathrm{r}=1 / 3$ hard & $\mathrm{r}=1 / 3$ soft \\
\hline 1 & 0.10 & 0.252 & 0.16 & 0.328 \\
\hline 2 & 0.19 & 0.382 & 0.25 & 0.475 \\
\hline 4 & 0.25 & 0.469 & 0.31 & 0.564 \\
\hline
\end{tabular}

TABLE III

BE of a DS/CDMA System with CBPSK and Channel Coding For MRC Diversity

\begin{tabular}{c|c|c|c|c}
\hline \hline $\mathrm{M}$ & $\mathrm{r}=1 / 2$ hard & $\mathrm{r}=1 / 2$ soft & $\mathrm{r}=1 / 3$ hard & $\mathrm{r}=1 / 3$ soft \\
\hline 1 & 0.14 & 0.346 & 0.21 & 0.449 \\
\hline 2 & 0.29 & 0.567 & 0.38 & 0.693 \\
\hline 4 & 0.39 & 0.716 & 0.49 & 0.858 \\
\hline
\end{tabular}

\section{CONCLUSIONS}

In summary, novel closed analytical expressions have been derived for the mean BER of a $p$-antenna postdetection selection diversity and MRC diversity DS/CDMA cellular system under an ideal averaged power control scheme and under any generic Rayleigh multipath environment. It has also been obtained tight upper bounds on the BER in terms of the cutoff rate for such diversity schemes when convolutional codes and soft decisions are employed. These expressions, the preciseness of which has been evaluated through simulations, have allowed us to assess the system performance and compare the gain in capacity for any of the analyzed schemes in the presence of a realistic environment.

\section{APPENDIX A}

To simplify the computation of

$$
\begin{aligned}
P_{e}= & \frac{1}{2} E[\operatorname{erfc}(\sqrt{\gamma})] \\
= & \frac{1}{2} \int_{0}^{\infty} \operatorname{erfc}(\sqrt{\gamma}) \sum_{i=1}^{P} \sum_{n=0}^{M-1} \pi_{n i}\left[1-\exp \left(-\frac{\gamma}{\bar{\gamma}_{n i}}\right)\right] \\
& \cdot \sum_{k=0}^{M-1} \frac{\pi_{k i}}{\bar{\gamma}_{k i}} \exp \left(-\frac{\gamma}{\bar{\gamma}_{k i}}\right) d \gamma
\end{aligned}
$$

let us make the change of variable $x=\sqrt{\gamma}$ so that

$$
\begin{aligned}
P_{e}= & \int_{0}^{\infty} \operatorname{erfc}(x) \sum_{i=1}^{P} \sum_{n=0}^{M-1} \pi_{n i}\left[1-\exp \left(-\frac{x^{2}}{\bar{\gamma}_{n i}}\right)\right] \\
& \cdot \sum_{k=0}^{M-1} \frac{\pi_{k i}}{\bar{\gamma}_{k i}} \exp \left(-\frac{x^{2}}{\bar{\gamma}_{k i}}\right) x d x \\
= & \frac{1}{2} \sum_{i=1}^{P} \sum_{n=0}^{M-1} \sum_{k=0}^{M-1} \frac{\pi_{n i} \pi_{k i}}{\bar{\gamma}_{k i}} \\
& \cdot\left[\int_{0}^{\infty} 2 x \operatorname{erfc}(x) \exp \left(-\frac{x^{2}}{\bar{\gamma}_{k i}}\right) d x\right. \\
& \left.-\int_{0}^{\infty} 2 x \operatorname{erfc}(x) \exp \left(-\frac{x^{2}}{\bar{\gamma}_{k n i}}\right) d x\right]
\end{aligned}
$$

with

$$
\bar{\gamma}_{k n i}=\frac{\bar{\gamma}_{k i} \bar{\gamma}_{n i}}{\bar{\gamma}_{k i}+\bar{\gamma}_{n i}} .
$$

If the two integrals in (31) are computed by parts, the expression of the mean BER obtained is given by

$$
\begin{aligned}
P_{e}= & \frac{1}{2} \sum_{i=1}^{P} \sum_{n=0}^{M-1} \sum_{k=0}^{M-1} \pi_{n i} \pi_{k i} \\
\cdot & {\left[\left(1-\sqrt{\frac{\bar{\gamma}_{k i}}{1+\bar{\gamma}_{k i}}}\right)-\frac{\bar{\gamma}_{k n i}}{\bar{\gamma}_{k i}}\left(1-\sqrt{\frac{\bar{\gamma}_{k n i}}{1+\bar{\gamma}_{k n i}}}\right)\right] }
\end{aligned}
$$

and after substituting (32) in (33), the final expression is

$$
\begin{aligned}
P_{e}= & \frac{1}{2} \sum_{i=1}^{P} \sum_{n=0}^{M-1} \sum_{k=0}^{M-1} \pi_{n i} \pi_{k i} \\
& \cdot\left[\frac{\bar{\gamma}_{k i}}{\bar{\gamma}_{k i}+\bar{\gamma}_{n i}}+\frac{\bar{\gamma}_{n i}}{\bar{\gamma}_{k i}+\bar{\gamma}_{n i}}\right. \\
& \left.\cdot \sqrt{\frac{\bar{\gamma}_{k i} \bar{\gamma}_{n i}}{\bar{\gamma}_{k i}+\bar{\gamma}_{n i}+\bar{\gamma}_{k i} \bar{\gamma}_{n i}}}-\sqrt{\frac{\bar{\gamma}_{k i}}{1+\bar{\gamma}_{k i}}}\right] .
\end{aligned}
$$

\section{APPENDIX B}

To simplify the computation of

$$
\begin{aligned}
P_{e}= & \frac{1}{2} E[\operatorname{erfc}(\sqrt{\gamma})] \\
= & \frac{1}{2} \int_{0}^{\infty} \operatorname{erfc}(\sqrt{\gamma}) \sum_{n=0}^{M-1} \pi_{n} \\
& \cdot\left(\frac{\gamma}{\bar{\gamma}_{n}^{2}}-\frac{2}{\bar{\gamma}_{n}} \sum_{i \neq n} \frac{\bar{\gamma}_{i}}{\bar{\gamma}_{n}-\bar{\gamma}_{i}}\right) \exp \left(-\frac{\gamma}{\bar{\gamma}_{n}}\right) d \gamma
\end{aligned}
$$


let us make the change of variable $x=\sqrt{\gamma}$ so that

$$
\begin{aligned}
P_{e}= & \int_{0}^{\infty} \operatorname{erfc}(x) \sum_{n=0}^{M-1} \pi_{n}\left(\frac{x^{3}}{\bar{\gamma}_{n}^{2}}-\frac{2 x}{\bar{\gamma}_{n}} \sum_{i \neq n} \frac{\bar{\gamma}_{i}}{\bar{\gamma}_{n}-\bar{\gamma}_{i}}\right) \\
& \cdot \exp \left(-\frac{x^{2}}{\bar{\gamma}_{n}}\right) d x \\
= & \sum_{n=0}^{M-1} \pi_{n}\left[\frac{1}{\bar{\gamma}_{n}^{2}} \int_{0}^{\infty} x^{3} \operatorname{erfc}(x) \exp \left(-\frac{x^{2}}{\bar{\gamma}_{n}}\right) d x\right. \\
& -\frac{2}{\bar{\gamma}_{n}} \sum_{i \neq n} \frac{\bar{\gamma}_{i}}{\bar{\gamma}_{n}-\bar{\gamma}_{i}} \int_{0}^{\infty} x \operatorname{erfc}(x) \\
& \left.\cdot \exp \left(-\frac{x^{2}}{\bar{\gamma}_{n}}\right) d x\right] .
\end{aligned}
$$

It is convenient to compute the integrals in (36) by parts, so it is straightforward to obtain the expression of the mean BER

$$
\begin{aligned}
P_{e}=\sum_{n=0}^{M-1} \pi_{n} & {\left[\left(\frac{1-\mu_{n}}{2}\right)^{2}\left(2+\mu_{n}\right)\right.} \\
& \left.-\left(1-\mu_{n}\right) \sum_{i \neq n} \frac{\bar{\gamma}_{i}}{\bar{\gamma}_{n}-\bar{\gamma}_{i}}\right]
\end{aligned}
$$

where

$$
\mu_{n}=\sqrt{\frac{\bar{\gamma}_{n}}{1+\bar{\gamma}_{n}}}
$$

\section{APPENDIX C}

When a Rake receiver is employed, the parameter $D(\lambda)$ can be expressed as [9, vol. II, p. 50]

$$
D(\lambda)=E\left\{\exp \left[-\sum_{n=0}^{M-1} \alpha_{n}^{2}\left(2 \lambda \sqrt{E_{b}}-\lambda^{2} I\right)\right]\right\}
$$

Let us define

$$
\lambda=s \frac{\sqrt{E_{b}}}{I}
$$

and notice that the signal-to-interference ratio at the output of the Rake receiver is

$$
\gamma_{b}=\sum_{n=0}^{M-1} \alpha_{n}^{2} \frac{E_{b}}{I}
$$

so (39) can be transformed in

$$
D(s)=E\left\{\exp \left[-\gamma_{b}\left(2 s-s^{2}\right)\right]\right\} .
$$

To compute this average, we need the expression of the pdf of $\gamma_{b}$ given by (7), so that

$$
\begin{aligned}
D(s)=\int_{0}^{\infty} e^{-\gamma_{b}\left(2 s-s^{2}\right)}\{ & P \sum_{n=0}^{M-1} \pi_{n}\left[1-\exp \left(-\frac{\gamma_{b}}{\bar{\gamma}_{n}}\right)\right] \\
& \left.\cdot \sum_{k=0}^{M-1} \frac{\pi_{k}}{\bar{\gamma}_{k}} \exp \left(-\frac{\gamma_{b}}{\bar{\gamma}_{k}}\right)\right\} d \gamma_{b}
\end{aligned}
$$

$$
\begin{aligned}
=P \sum_{n=0}^{M-1} \sum_{k=0}^{M-1} \pi_{n} \pi_{k} & {\left[\frac{1}{1+\bar{\gamma}_{k}\left(2 s-s^{2}\right)}\right.} \\
& \left.-\frac{\bar{\gamma}_{n}}{\bar{\gamma}_{n}+\bar{\gamma}_{k}+\bar{\gamma}_{n} \bar{\gamma}_{k}\left(2 s-s^{2}\right)}\right]
\end{aligned}
$$

Then, minimizing the parameter $D(s)$ expressed in (43), the following expression is obtained:

$$
\begin{aligned}
D & =\min _{s \geq 0} D(s) \\
& =P \sum_{n=0}^{M-1} \sum_{k=0}^{M-1} \pi_{n} \pi_{k}\left[\frac{1}{1+\bar{\gamma}_{k}}-\frac{\bar{\gamma}_{n}}{\bar{\gamma}_{n}+\bar{\gamma}_{k}+\bar{\gamma}_{n} \bar{\gamma}_{k}}\right] .
\end{aligned}
$$

\section{APPENDIX D}

When MRC is selected as form of diversity, from (42) we have that

$$
\begin{aligned}
D(s)= & \int_{0}^{\infty} e^{-\gamma_{b}\left(2 s-s^{2}\right)}\left[\sum_{n=0}^{M-1}\right. \\
& \left.\cdot \pi_{n}\left(\frac{\gamma_{b}}{\bar{\gamma}_{n}^{2}}-\frac{2}{\bar{\gamma}_{n}} \sum_{i \neq n} \frac{\bar{\gamma}_{i}}{\bar{\gamma}_{n}-\bar{\gamma}_{i}}\right) \exp \left(-\frac{\gamma_{b}}{\bar{\gamma}_{k}}\right)\right] d \gamma_{b} \\
= & \prod_{n=0}^{M-1}\left[\frac{1}{1+2 \bar{\gamma}_{n}\left(2 s-s^{2}\right)}\right] .
\end{aligned}
$$

Therefore, the $D$ parameter is given by

$$
D=\min _{s \geq 0} D(s)=\prod_{n=0}^{M-1}\left(\frac{1}{1+\bar{\gamma}_{n}}\right)^{2} .
$$

\section{REFERENCES}

[1] F. Simpson and J. M. Holtzman, "Direct sequence CDMA power control, interleaving and coding," IEEE J. Select. Areas Commun., vol. 11, no. 7, pp. 1085-1095, 1993.

[2] S. Ariyavisitakul and L. F. Chang, "Signal and interference statistics of a CDMA system with feedback power control," IEEE Trans. Commun., vol. 41, no. 11, pp. 1626-1634, 1993.

[3] M. J. McTiffin, A. P. Hulbert, T. J. Ketseoglou, W. Heimsch, and G. Crisp, "Mobile access to an ATM network using a CDMA air interface," IEEE J. Select. Areas Commun., vol. 12, no. 5, pp. 900-908, 1994.

[4] A. J. Rustako, Jr., Y. S. Yeh, R. R. Murray, "Performance of feedback and switch space diversity $900 \mathrm{MHz}$ FM mobile radio systems with Rayleigh fading," IEEE Trans. Veh. Technol., vol. VT-22, pp. 173-184, Nov. 1973.

[5] G. Femenías and R. Agustí, "Switched-diversity trellis-coded 8-DPSK for mobile radio applications," in 40th IEEE Vehicular Technology Conf., Orlando, FL, May 1990, pp. 476-481.

[6] J. G. Proakis, Ed. Digital Communication. New York: McGraw-Hill, 1983.

[7] A. J. Viterbi, "Spread spectrum communications-Myths and realities," IEEE Commun. Mag., vol. 17, pp. 11-18, May 1979.

[8] J. K. Omura and B. K. Levitt, "Coded error probability evaluation for antijam communication systems," IEEE Trans. Commun., vol. COM-30, pp. 896-903, May 1982.

[9] M. K. Simon, J. K. Omura, R. A. Scholtz, and B. K. Levitt, Eds., Spread Spectrum Communications. Rockville, MD: Computer Science, 1985.

[10] M. Kavehrad and P. J. McLane, "Performance of low-complexity channel coding and diversity for spread spectrum in indoor wireless communications," AT\&T Tech. J., vol. 64, no. 8, pp. 1927-1965, 1985.

[11] Cost 207, "Digital land mobile radio communications," Information Technologies and Sciences, Commission of the European Communities, 1989.

[12] G. R. Cooper and R. W. Nettleton, "Spectral efficiency in cellular landmobile communications: A spread-spectrum approach," Purdue Univ., West Lafayette, IN, Final Rep. 15-5-1977/31-12-1978, TR-EE 78-44. 


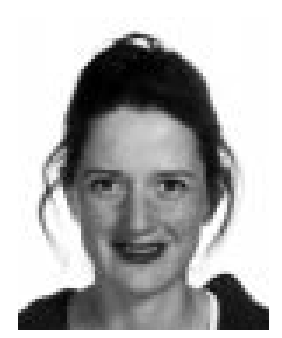

Pilar Díaz (M'94) was born in Barcelona, Spain, on November 29, 1967. She received the Engineer and Doctor Engineer degrees in telecommunication engineering from the Universitat Politècnica de Catalunya (UPC), Spain, in 1990 and 1994, respectively.

In 1990, she joined the Escola Tècnica Superior d'Enginyers de Telecomunicació de Barcelona, Spain, where she became an Assistant Professor in 1991 and an Associate Professor in 1995. She has been working in the field of digital radio communications with particular emphasis on personal, indoor, and mobile communications. Her main research interests lie in the area of radio multiple access and spread-spectrum systems, both FH and DS/CDMA systems. She participated in the RACE Program and Cost European Action, and at present, she is actively participating in the ACTS European Research Program.

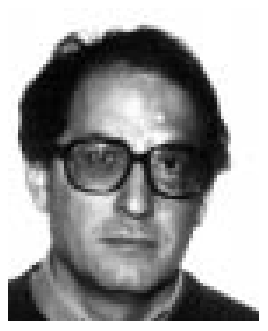

Ramón Agustí (M'78) was born in Riba-roja d'Ebre, Spain, on August 15, 1951. He received the Engineer of Telecommunication degree from the Universidad Politècnica de Madrid, Spain, in 1973 and the Ph.D. degree from the Universitat Politècnica de Catalunya (UPC), Spain, in 1978.

In 1973, he joined the Escola Tècnica Superior d'Enginyers de Telecomunicació de Barcelona Spain, where he became Full Professor in 1987

$\mathrm{He}$ has been working in the field of digital communications with particular emphasis on digital radio, both fixed radio relay and mobile communications. He has also been concerned with the performance analysis and development of frequencyhopped spread-spectrum systems. He participated in the COST 231 and RACE European research programs and currently is participating in the ACTS program. His research interests are in the area of mobile communications with special emphasis on CDMA systems and packet radio networks. 